\title{
Advances in Imaging in Age-Related Macular Degeneration
}

\author{
Pearse A. Keane $\cdot$ Dawn A. Sim $\cdot$ Srinivas R. Sadda
}

Published online: 2 February 2013

(C) Springer Science + Business Media New York 2013

\begin{abstract}
Age-related macular degeneration (AMD) is the leading cause of severe visual loss in people aged 50 years or older in the developed world. Advances in ocular imaging have driven many of the major breakthroughs in our understanding of the disease's pathogenesis, as well as many improvements in diagnosis and treatment. In this review, we describe the latest advances in ocular imaging applicable to patients with AMD. We begin with an overview of topographic imaging techniques, with an emphasis on emerging technologies such as ultrawidefield imaging, fundus autofluorescence, and adaptive optics. We next describe the latest advances in optical coherence tomography imaging, with an emphasis on novel image acquisition and analysis techniques of use for patients with AMD. In both cases, we describe the current state of the art for clinical imaging of patients with AMD and highlight new approaches likely to appear in clinical settings in the near future.
\end{abstract}

Keywords Age-related macular degeneration - Optical coherence tomography · Imaging · Angiography ·

Autofluorescence

\begin{abstract}
Abbreviations
AMD Age-related macular degeneration

$\mathrm{CNV}$ Choroidal neovascularization
\end{abstract}

\section{P. A. Keane - D. A. Sim}

NIHR Biomedical Research Centre for Ophthalmology, Moorfields Eye Hospital NHS Foundation Trust and UCL Institute of Ophthalmology, London, UK

\section{S. R. Sadda $(\square)$}

Doheny Eye Institute-DEI 3623, Keck School of Medicine of the University of Southern California, 1450 San Pablo Street, Los Angeles, CA 90033, USA

e-mail: ssadda@doheny.org

$\begin{array}{ll}\text { OCT } & \text { Optical coherence tomography } \\ \text { SLO } & \text { Scanning laser ophthalmoscope } \\ \text { CCD } & \text { Charge-coupled device } \\ \text { HRA } & \text { Heidelberg retinal angiograph } \\ \text { HRT } & \text { Heidelberg retinal tomograph } \\ \text { FA } & \text { Fluorescein angiography } \\ \text { TAG } & \text { Topographic angiography } \\ \text { RLA } & \text { Retinal leakage analyzer } \\ \text { ICG } & \text { Indocyanine green } \\ \text { PCV } & \text { Polypoidal choroidal vasculopathy } \\ \text { RAP } & \text { Retinal angiomatous proliferation } \\ \text { FAF } & \text { Fundus autofluorescence } \\ \text { MPOD } & \text { Macular pigment optical density } \\ \text { ETDRS } & \text { Early Treatment of Diabetic Retinopathy Study } \\ \text { EDI } & \text { Enhanced depth imaging } \\ \text { AREDS } & \text { Age-Related Eye Disease Study }\end{array}$

\section{Introduction}

Age-related macular degeneration (AMD), if untreated, is a leading cause of irreversible visual loss in older populations [1]. At various points in the disease's history, crucial breakthroughs have been made in our understanding of its pathogenesis, leading to significant improvements in diagnosis and treatment. In many cases, these paradigm shifts have flowed directly from advances in ocular imaging technology [2]. The first such transformative imaging advance was the invention of fundus fluorescein angiography by Novotny and Alvis [3]. Using this method, Gass recognized the central role of choroidal neovascularization (CNV) in the pathophysiology of neovascular AMD (Fig. 1), laying the foundation for treatment of the disease using laser 


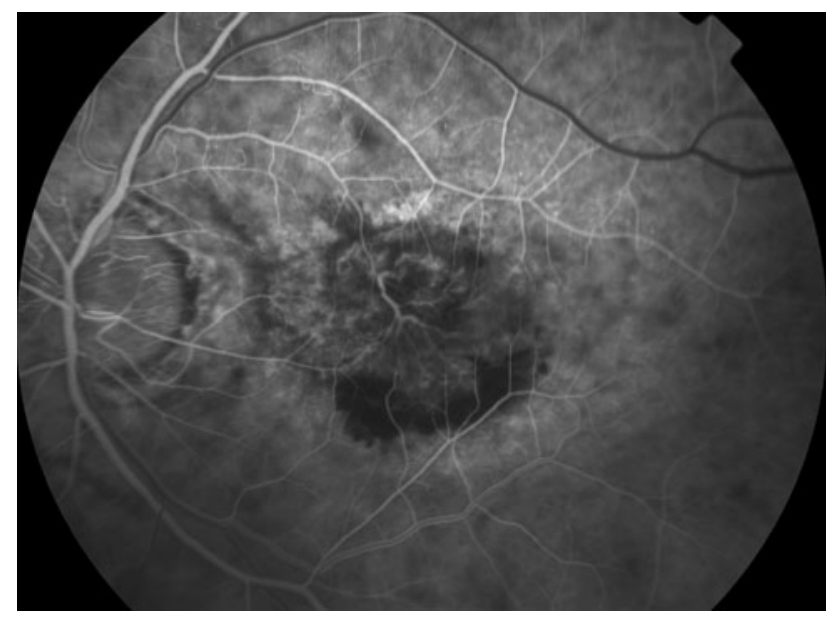

Fig. 1 Early phase fluorescein angiographic image demonstrating choroidal neovascularization $(\mathrm{CNV})$ in a patient with neovascular age-related macular degeneration (AMD)

photocoagulation $[4,5]$. The subsequent clinical trial of this therapeutic approach — the Macular Photocoagulation Study (MPS) - greatly enhanced our knowledge of disease features and natural history [6]. In the 1980 and 1990s, another imaging modality - color fundus photography-played a crucial role in major epidemiological studies of AMD (e.g., Beaver Dam Eye Study, Blue Mountains Eye Study, Rotterdam Eye Study), establishing and clarifying major risk factors for the disease [7]. More recently, the advent of a wholly new imaging modality, optical coherence tomography (OCT), and the introduction of effective pharmacotherapies, has ushered in a new era in disease management $[8 \bullet \bullet]$.

In this review, we describe recent advances in imaging for patients with AMD. We divide these advances into two main categories. Firstly, we provide an overview of topographic imaging techniques (i.e., the use of fundus cameras and/or scanning laser ophthalmoscopes (SLO) to obtain two-dimensional images of the ocular fundus), and how these techniques have been modified to greatly extend their application. Secondly, we describe the latest advances in tomographic imaging techniques (i.e., the use of OCT to provide cross-sectional and three-dimensional views of the retina and underlying structures). In both cases, we describe the current state of the art for clinical imaging of patients with AMD and highlight new approaches likely to appear in clinical settings in the near future.

\section{Topographic Imaging}

\section{Color Fundus Photography}

The introduction of the ophthalmoscope by von Helmholtz in 1851 allowed physicians to visualize the ocular fundus for the first time in living human subjects [9]. Around this period, significant advances were also made in the nascent field of photography and, by the end of the nineteenth century, the first fundus photographic images had been obtained [10]. It was not until the 1950s, however, with the advent of electronic flashes and 35-mm cameras, that the field of modern fundus photography truly emerged [11••]. Since this time, fundus cameras have undergone substantial refinements, including optimization for nonmydriatic and stereoscopic image acquisition, and transition from analog to digital image capture [12]. Efforts are also underway, driven in large part by the widespread adoption of smartphone photography [13], to make fundus cameras more portable and easy to use. At present, retinal cameras are typically described by their optical field of view, with an angle of $30^{\circ}$ or $35^{\circ}$ most commonly used (creating images with a magnification of 2.5 and 2.45 respectively) [12]. In clinical practice, commonly used fundus cameras include the Topcon TRC-50DX (Topcon, Japan) and the Zeiss FF450 ${ }^{\text {plus }}$. In patients with AMD, color fundus photography remains a crucial component of clinical trials and epidemiological studies, with the Age-Related Eye Disease Study 2 (AREDS 2) a leading recent example [14].

\section{Scanning Laser Ophthalmoscopy}

Fundus photography is dependent on the use of a bright ring of white light to illuminate the ocular fundus. The light reflected from the fundus is then projected onto the pixel array of a charge-coupled device (CCD), and all the pixels of the image are created in the same instant [10]. In the 1980s, the introduction of SLO devices provided an alternative method for the acquisition of fundus images [15]. SLO devices employ a single point of laser light, at a specific wavelength, which is scanned across the retina in a raster pattern (i.e., a series of parallel horizontal lines). As a result, images are created one pixel at a time. As only a small area of the fundus is illuminated at any one time, the effects of light scatter are reduced; thus, SLO devices are capable of producing fundus images with higher contrast than standard fundus cameras (fundus cameras typically have higher temporal and spatial resolution) [11••]. In clinical practice, commonly used SLO instruments include the Heidelberg Retina Angiograph-2 (HRA-2) (Heidelberg Engineering, Germany) and the Nidek F-10 (Nidek, Japan).

SLO systems also employ a confocal aperture, so that only light returning from a specific plane of interest is used to reconstruct the fundus image $\left[11^{\bullet \bullet}\right]$. This feature allows acquisition of multiple images at different focal planes (i.e., the device can create a "stack" of images from different levels of the retina and choroid). Three-dimensional reconstruction of these images then allows tomographic (cross-sectional) visualization of the ocular fundus. 
Fig. 2 Near-infrared reflectance image (left) and blue light fundus autofluorescent image (right) demonstrating drusen and geographic atrophy in a patient with dry age-related macular degeneration (AMD)
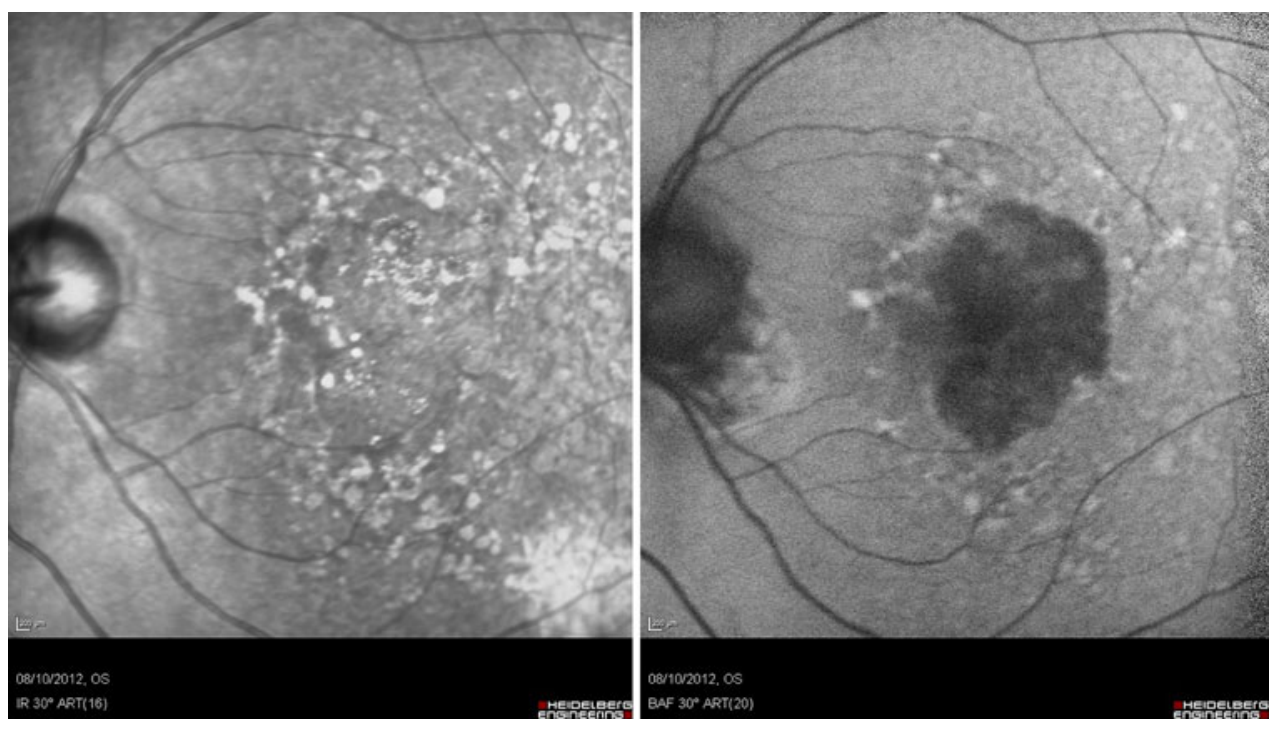

Although the axial resolution of this method is inferior to that of OCT (see below), this method has proven useful for assessment of the optic nerve head in patients with glaucoma. In clinical practice, commonly used devices include the Heidelberg retina tomograph (HRT) (Heidelberg Engineering, Germany) [16].

A more recent, novel, modification of SLO systems is the introduction of "retro-mode" imaging [17]. In this technique, the confocal aperture of the SLO is decentered to one side. As a result, light directly reflected from a lesion of interest is blocked from the image. Instead, only laterally reflected light from one side of the lesion contributes to image formation. For example, if a single druse is focused on, light laterally reflected from one side of the druse will be seen. The other side of the druse, whose reflected light is not captured, appears as a dark "shadow", producing a pseudo-three dimensional image effect. Use of retro-mode SLO imaging may enhance the visualization of drusen and retinal pigment epithelium (RPE) abnormalities in patients with AMD; however, in the age of OCT, its exact clinical role remains unclear $[17,18]$.

\section{Monochromatic Imaging}

As light penetration through the retina is dependent on its wavelength, the incorporation of monochromatic light filters in fundus photography has aided visualization of many anatomic structures and disease features [10]. Longer wavelengths of light penetrate more deeply through the retina; the use of near-infrared filters has thus greatly improved visualization of subretinal and choroidal structures (Fig. 2). Conversely, the use of shorter wavelengths of light-e.g., blue light-allows improved imaging of superficial retinal structures such as the retinal nerve fiber layer and epiretinal membranes. Intermediate wavelengths of light-e.g., green light ("red-free")—provide optimal fundus images for general purposes, being reflected by the RPE but absorbed by hemorrhage.

SLO-based fundus imaging is particularly well suited to monochromatic image acquisition, as it typically employs laser light sources at a number of fixed wavelengths. For example, confocal blue light reflectance (CBR) has also been used in the evaluation of patients with idiopathic macular telangiectasia (MacTel) and in the non-invasive assessment of eyes with retinal capillary non-perfusion [19, 20]. The HRA-2 system now incorporates a "multi-color" mode, where separate fundal images are obtained with blue, green, and near-infrared filters, and which can then be examined individually or in combination. Similarly, the Optos P200 widefield imaging system (Optos plc., Scotland) uses an SLO to acquire fundus images at two distinct light wavelengths, which are then combined in a single composite pseudo-color image. The clinical utility of "multi-color" imaging in AMD remains unclear, but may be of particular use in aiding visualization of certain forms of drusen (e.g., reticular pseudodrusen), and for aiding visualization of geographic atrophy and choroidal disease.

\section{Fundus Angiography}

In 1961, Novotny and Alvis reported the first demonstration of fluorescein angiography (FA). They modified a standard fundus camera with monochromatic light filters and then obtained a series a fundus images following intravenous administration of a fluorescent contrast agent (sodium fluorescein) [3]. This technique allowed greatly enhanced visualization of the retinal vasculature as well as assessment of its structural integrity. SLO systems are also well suited to angiographic imaging. Planimetric measurements can easily be obtained from FA image sets [21]. 
In patients with AMD, this has proven particularly useful in clinical trials of new therapeutics, where measurements of $\mathrm{CNV}$ leakage area and lesion size can easily be obtained [22]. In more recent years, efforts have also been made to obtain direct measurements of fluorescein leakage. Examples of this approach include "topographic angiography" (TAG) and the "retinal leakage analyzer" (RLA) [23, 24]. In both cases, a confocal SLO is used to obtain a stack of 32 images at different focal planes between the vitreous and the choroid. The images are then aligned and fluorescence intensity profiles generated.

Indocyanine green (ICG) is a fluorescent dye with peak excitation and emission spectra in near-infrared wavelengths [25]. As long-wavelength monochromatic light has enhanced depth penetration (see above), ICG angiography is particularly well suited to visualization of the choroidal vasculature and of structures otherwise obscured by retinal hemorrhage. Relative to sodium fluorescein, a greater percentage of ICG is bound to albumin and other serum proteins; this characteristic further aids choroidal vascular mapping (the escape of unbound sodium fluorescein from the fenestrated capillaries of the choriocapillaris-"choroidal flush"-quickly obscures the underlying vessels in conventional angiography). In patients with AMD, ICG angiography is most commonly used for the detection of polypoidal choroidal vasculopathy (PCV), but is also useful in identifying retinal angiomatous proliferation (RAP) and for uncovering $\mathrm{CNV}$ in eyes where the lesion is obscured by a serous pigment epithelial detachment or substantial amounts of submacular hemorrhage [26].

\section{Fundus Autofluorescence}

Following the widespread adoption of FA, it became apparent that certain areas of the fundus could be seen to fluoresce even prior to the injection of contrast. This phenomenon, termed "pseudofluorescence", was initially considered to be an artifact, and ascribed to overlap in the transmission wavelengths of the monochromatic light filters that were used [27]. However, it soon became clear that many parts of the fundus exhibit fluorescent properties when excited by specific wavelengths of light-so-called "fundus autofluorescence" (FAF) [28••].

Evaluation of FAF phenomena was initially challenging, due in large part to the low intensity of the autofluorescent signal, but also due to fluorescence originating from the crystalline lens [28••]. In the early 1990s, Delori et al. used a spectrophotometer to measure the excitation and emission spectra of FAF over small areas of the retina $\left(2^{\circ}\right)$ $[29,30]$. This important work demonstrated that the dominant source of FAF was lipofuscin, a byproduct of photoreceptor outer-segment processing that accumulates in RPE cells [31]. Subsequent use of SLO technology allowed
FAF imaging over larger retinal areas, with the use of a confocal aperture greatly reducing autofluorescence emanating from sources anterior to the retina [32]. In current clinical practice, the SLO device most commonly used for FAF imaging is the HRA-2, "Bluepeak" autofluorescence system (Heidelberg Engineering), with an excitation wavelength of $488 \mathrm{~nm}$ [33].

FAF imaging can also be performed using standard fundus cameras. In the absence of confocal optics, however, the detected fluorescence derives from all tissue levels in the light beam with fluorescent properties, a source of significant noise. This shortcoming is particularly evident when bluelight is used, as the lens contributes significantly to the signal (especially in older subjects with significant nuclear sclerosis of the lens). To address this, Spaide developed longer wavelength filters (excitation: $535-580 \mathrm{~nm}$ ) for use with a standard fundus camera (Spaide Autofluorescence Filters, Topcon) [28•, 34]. The images generated in this manner differ subtly from those obtained with blue-light. For example, with blue-light FAF, the fovea appears hypoautofluorescent due to the blocking effect of macular pigmentwith the longer wavelengths used in green-light FAF, this effect is less pronounced. This finding has been utilized in socalled "dual-wavelength" autofluorescence, where comparison of FAF images at $488 \mathrm{~nm}$ and at longer wavelengths is used to assess macular pigment optical density (MPOD) $[35,36]$. FAF imaging is thus clearly dependent on the wavelength of light used - for example, when near-infrared light $(787 \mathrm{~nm})$ is used for excitation, melanin replaces lipofuscin as the dominant fluorophore [37]. In recent years, such "near-infrared" autofluorescence has been used to investigate patients with inherited retinal degenerations and toxic retinopathies, although its role in AMD, if any, remains unclear [38, 39].

In general, blue- and green-light FAF imaging has proven valuable for assessment of patients with AMD, in particular those patients with geographic atrophy [34, 40, 41]. In such patients, areas of atrophy appear hypoautofluorescent due to the confluent loss of RPE cells (and thus loss of the fluorescent signal generated from their lipofuscin granules). The boundaries of these atrophic areas are often more clearly visualized on FAF imaging than on conventional fundus photography (Fig. 2) [42]. A number of studies have also suggested that the autofluorescent patterns seen in the areas surrounding foci of geographic atrophy can be predictive of the rate of disease progression $[40,43]$.

\section{Ultrawidefield Imaging}

Conventional fundus imaging, whether it be with a fundus camera or an SLO, typically utilizes a $30^{\circ}$ or $35^{\circ}$ field of view. While such an approach allows optimal visualization of the posterior pole (i.e., the area of the retina 
encompassing the optic disc and macula), much of the peripheral retina is not captured. Techniques such as sevenfield image acquisition have previously been developed to address this shortcoming [10]. However, even in optimal circumstances, this does not allow for complete coverage of the retina and is often technically challenging. Fundus contact lens have also been employed to afford wider fields of view (e.g., the Staurenghi lens), however, the need to apply the lens directly to the eye is a limiting factor in the widespread utilization of such approaches [44, 45]. Other contact widefield imaging devices include RetCam (Clarity Medical Systems, United States) and Panoret-1000 (Medibell Medical Vision Technologies, Israel) [46].

Significant advances have been made in recent years with the release non-contact ultrawidefield imaging systems such as the Optos 200 Tx system (Optos plc., Scotland) [47-49]. This system uses an SLO with two wavelengths of laser light [green $(532 \mathrm{~nm})$ and red $(633 \mathrm{~nm})$ ] to recreate a composite color image of the posterior segment. Through the use of a large ellipsoid mirror, images with a $200^{\circ}$ field of view can be obtained through an undilated pupil. In this manner, approximately $80 \%$ of the total retinal surface area can be visualized. By reducing the field of view to $100^{\circ}$ (ResMax mode), a $10-15 \%$ increase in resolution is achieved (but only covering $25 \%$ of the retinal area-a nonlinear reduction due to the nature of the image acquisition). A noncontact widefield $\left(100^{\circ}\right)$ imaging system has also been introduced recently by Heidelberg Engineering.

As with standard fundus cameras and other SLO systems, the Optos Ultrawidefield system can be modified to provide additional information. For example, the use of filters allows ultrawidefield autofluorescence and angiographic imaging [48, 50]. Ultrawidefield autofluorescence imaging may be of particular use for detection of degenerative changes of the peripheral retina in patients with AMD [51]. Such an approach may lead to improved phenotyping of the condition, a particularly important process as more is learnt about the disparate nature of the disease and its underlying genetic risk factors.

\section{Adaptive Optics}

The transverse optical resolution of fundus cameras and SLO devices is limited by the presence of defects, or aberrations, in the optical system of the eye (i.e., the cornea and lens) [52••]. Until the early 1990s, there existed no effective way to compensate for these defects. However, wavefront sensors originally developed for astronomical purposes have now been adapted for use in the eye, allowing real-time measurement of ocular aberrations. Once measured, the detected aberrations can be corrected (i.e., rendered flat) using deformable mirrors (mirrors with large numbers of small electronically controlled actuators on their rear surface that can push and pull the mirror within a range of $\pm 2 \mu \mathrm{m}$, allowing it to adopt any desired configuration). By incorporating wavefront sensing and correction into existing optical imaging platforms- "adaptive optics"-it is now possible to acquire images of the retina with cellular-level resolution, and in a noninvasive fashion.

Adaptive optics has been incorporated into both fundus cameras and confocal SLO systems [53, 54]. Adaptive optics "flood-illuminated" fundus cameras are now commercially available and approved for use in clinical settings (e.g., "rtx1 Adaptive Optics Retinal Camera", Imagine Eyes, France). While these devices offer greatly improved transverse resolution, their field of view is still limited (e.g., $4^{\circ} \times 4^{\circ}$ ). In contrast to flood-illuminated adaptive optics fundus cameras, adaptive optics SLOs use the same source of light for the wavefront sensor as for the retinal illumination-as a result, the wavefront sensor is able to measure aberrations from the entire scanned area of the retina. When imaging the retina, SLO devices provide a transverse resolution of approximately $15 \mu \mathrm{m}$, but are limited to an axial resolution of approximately $300 \mu \mathrm{m}$. With the addition of adaptive optics correction to an SLO, the transverse resolution may be increased to less than $3 \mu \mathrm{m}$, while the axial resolution may be improved to $40 \mu \mathrm{m}$.

Cone photoreceptors are the dominant feature seen with both flood-illuminated and SLO devices [52••]. Rods are smaller and less easily seen; however, recent advances, including the use of smaller confocal pinholes and improvements in registration algorithms, now allow clear images of single rods to be obtained in the living human eye (as well as aiding visualization of the smallest cones at the foveal center) [55]. Direct in vivo visualization of single RPE cells remains challenging. Unlike cone photoreceptors, the intrinsic contrast of RPE cells is poor. In addition, much of the light scattered by the RPE is masked by the overlying photoreceptors, which are also highly scattering. To date, direct imaging of the RPE has only been possible in human subjects with retinal disease where absence of cone photoreceptors has allowed visualization of the underlying RPE [56]. With its ability to provide direct visualization of individual photoreceptors, adaptive optics has already proven useful in the study of inherited retinal degenerations [57]. With advances in its field of view, and ease of use, it is likely to prove valuable in future study of geographic atrophy and drusen progression in patients with AMD.

\section{Tomographic Imaging}

Optical Coherence Tomography—Basic Principles

OCT is a cross-sectional (tomographic) ocular imaging method, analogous to ultrasonography, but which measures 
reflected light waves rather than sound waves [58••]. These measurements are achieved indirectly using low-coherence tomography, an approach first described by Huang et al. [59]. In this technique, the combination of light reflected from a tissue of interest, and light reflected from a reference path, produces characteristic patterns of light interference dependent on the mismatch between the reflected waves. Since the time delay and amplitude of one of the waves (i.e., the reference path) is known, the time delay and intensity of light returning from the sample tissue may then be extracted from the interference pattern. As the wavelength of light is so much shorter than that of sound, OCT imaging generates image sets with considerably superior resolution to those of ultrasonography (e.g., axial resolutions of 3-8 $\mu \mathrm{m}$ in commercially available systems). OCT has been widely adopted for the management of vitreoretinal disorders, and nowhere more so than for the evaluation of AMD, a change driven in large part by the need for frequent anti-angiogenic therapy in patients with neovascular AMD [60••].

\section{Spectral Domain OCT—Raster Scanning}

In the original OCT systems, the interference patterns generated were varied as a function of time using a moving mirror in the reference pathway; such devices were commonly referred to as "time domain" OCT. In more recent OCT devices, the interference patterns generated are measured as a function of frequency, using a spectrometer $[58 \bullet \bullet, 61]$. These "spectral domain" OCT devices remove the need for a moving reference mirror and thus facilitate greatly enhanced image acquisition speed. For example, time domain OCT systems (e.g., Stratus OCT, Carl Zeiss Meditec, Germany) acquire images at $400 \mathrm{~A}$-scans per second, while spectral domain OCT systems (e.g., 3D OCT, Topcon; Cirrus HD-OCT, Carl Zeiss Meditec; Spectralis OCT, Heidelberg Engineering) typically acquire images at speeds in excess of 20,000 A-scans per second. As a result, greater sampling of the macula is possible for any given image set (e.g., 128 line scans in a horizontal raster pattern), and areas of focal pathology are less likely to be missed. In patients with neovascular AMD, this feature is of crucial importance, as small pockets of intra- or subretinal fluid may be an early sign of disease activity and trigger the need for retreatment with anti-angiogenic injections [62].

\section{Spectral Domain OCT_-OCT Fundus Images}

The ability to perform dense raster scanning also allows spectral domain OCT systems to generate two-dimensional representations of the scanned macular area- "OCT fundus images" or "projection images" (Fig. 3) [63]. These images are generated from dense raster scan image sets by summing

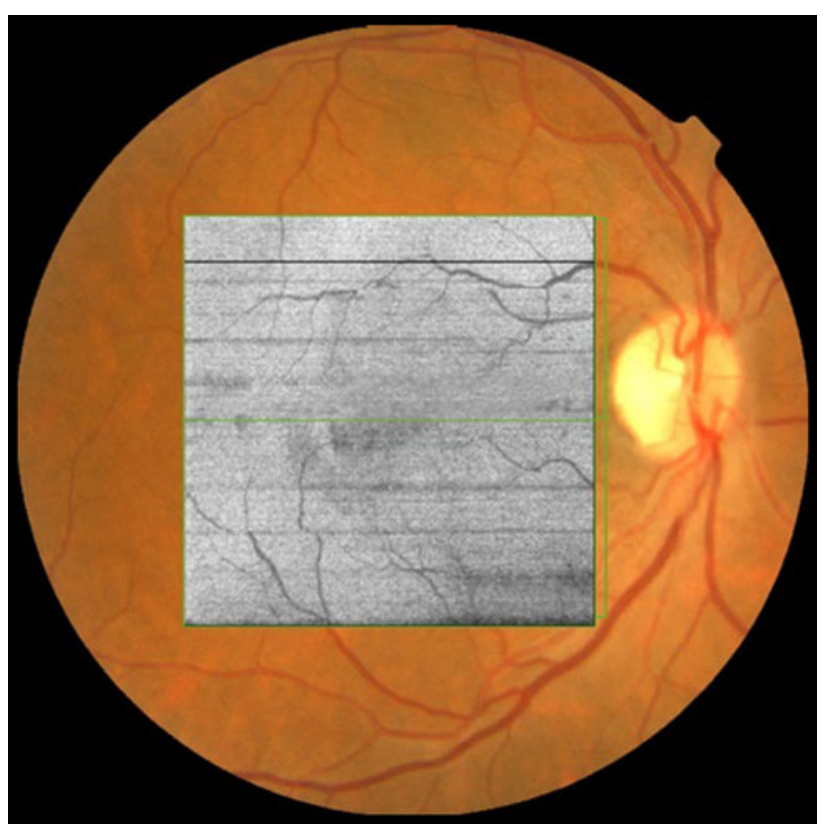

Fig. 3 "OCT fundus image" superimposed on color fundus photography of a right eye. These images are generated from dense raster scan image sets by summing the intensity of pixels for each individual A-scan and allow visualization of the retinal vasculature which is spatially consistent with that seen on color photography

the intensity of pixels for each individual A-scan. OCT fundus images show a direct view of the macula in which the retinal vascular arcades may be clearly visible and spatially consistent with the vasculature on color photographic, or angiographic, images. OCT fundus images are useful in the clinical setting as they can facilitate registration of any point on an OCT image with a corresponding point on the retinal surface, and the acquisition of images at the same location over time. These images are particularly important in patients with advanced AMD, as they show an increased total signal in areas where geographic atrophy is apparent on clinical examination (due to increased penetration of light into the choroid where atrophy of the RPE and choriocapillaris occurs). Measurements of geographic atrophy taken from OCT fundus images are highly reproducible and correlate well with those obtained from other modalities such as fundus photography and autofluorescence [64, 65]. As a result, OCT is increasingly being advocated as a method for measuring geographic atrophy progression in patients with AMD (for example, Cirrus HD-OCT, Carl Zeiss Meditec, now incorporates software that uses this approach for tracking of geographic atrophy progression).

\section{Spectral Domain OCT—RPE Elevation Mapping}

Since its introduction, efforts have been made to obtain quantitative measurements from OCT images in an automated fashion, and thus provide objective measures of 
Fig. 4 Optical coherence tomography (OCT) B-scan (3D OCT-2000, Topcon, Japan) obtained from a patient with large numbers of drusen. Automated segmentation of the retinal pigment epithelium (RPE) facilitates quantitative analyses of drusen and pigment epithelium detachment (PED)

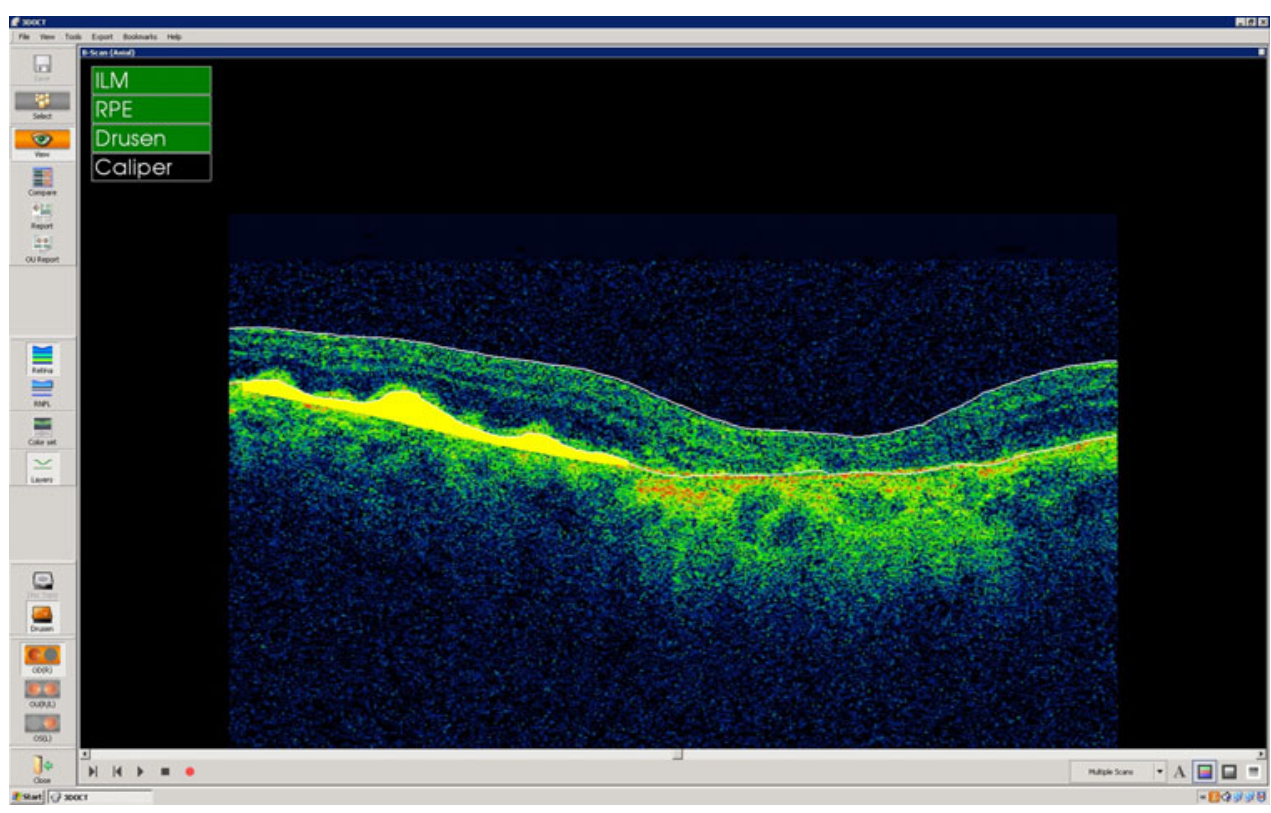

disease progression and/or response to therapy [66]. Until recently, such efforts have focused on determination of retinal thickness via automated delineation (segmentation) of the inner and outer retinal boundaries. Using this approach, it is possible to measure retinal thickness at multiple locations and to construct retinal thickness maps corresponding to the Early Treatment of Diabetic Retinopathy Study (ETDRS) subfields [67]. In recent years, these segmentation results have also been used to perform more detailed analyses in patients with AMD. In particular, segmentation of the RPE can be used to derive information about its topography (Fig. 4) i.e., generate RPE elevation maps-quantitative difference maps between the segmented RPE and an interpolated RPE floor [68]. These RPE elevation maps can thus be used to provide information regarding number, area, and volume of drusen in patients with dry AMD, as well as quantification of pigment epithelium detachment (PED) in patients with neovascular AMD and other disorders [69]. Such software is now a standard feature of many commercially available OCT systems (e.g., "Drusen Analysis", 3D OCT 2000, Topcon; "RPE Elevation Analysis", Cirrus HDOCT, Carl Zeiss Meditec) (Fig. 5). Efforts are currently underway to perform automated characterization of PEDs (fibrovascular versus drusenoid versus serous) in patients with AMD, through the use of OCT [70].

\section{Spectral Domain OCT-Choroidal Scanning Protocols}

With spectral domain OCT systems, visualization of the choroid is now possible using specialized scanning protocols_-so-called "enhanced depth imaging" (EDI) OCT [71]. In this approach, the rapid image acquisition speed of spectral domain OCT, in combination with real-time eye- tracking, is used to facilitate multiple B-scan averaging, i.e., each OCT B-scan in any given image set is constructed from the average of many individual B-scans acquired in an identical location. By further adjusting the point of maximum imaging sensitivity, high quality images of the choroid can be readily obtained (Fig. 6). EDI-OCT is most closely associated with the Spectralis system, however, choroidal visualization is also possible with other devices using alternate methods (e.g., "selective pixel profiling", Cirrus HDOCT, Carl Zeiss Meditec) [72]. Evaluation of the choroid in this manner may be of particular interest in the elderly populations at risk of AMD, with the phenotype of "agerelated choroidal atrophy" recently described [73, 74].

\section{Swept Source/Long Wavelength OCT}

As described above, spectral domain OCT devices analyze interference patterns as a function of frequency through the use of a spectrometer (light waves entering the spectrometer are first dispersed using a diffraction grating and then analyzed using a charge-coupled device (CCD) camera) [58 • $]$. An alternative approach to the assessment of interference patterns in this way is through the use of a frequency-swept ("tunable") laser light source-so-called "swept source" OCT [75]. The next generation of commercial OCT systems is likely to employ such technologies, with early examples including the DRI OCT-1, Topcon, for retinal imaging and the CASIA, OCT SS-1000, Tomey, for anterior segment imaging. Swept source OCT offers the potential for further increases in image acquisition speed (100,000 A-scans per second for DRI OCT-1, but much higher for research prototype devices). These increases may facilitate scanning of larger areas (e.g., 
Fig. 5 Detailed quantitative analyses of drusen obtaining using optical coherence tomography (OCT) in a patient with dry age-related macular degeneration (AMD) (3D OCT-2000, Topcon, Japan)

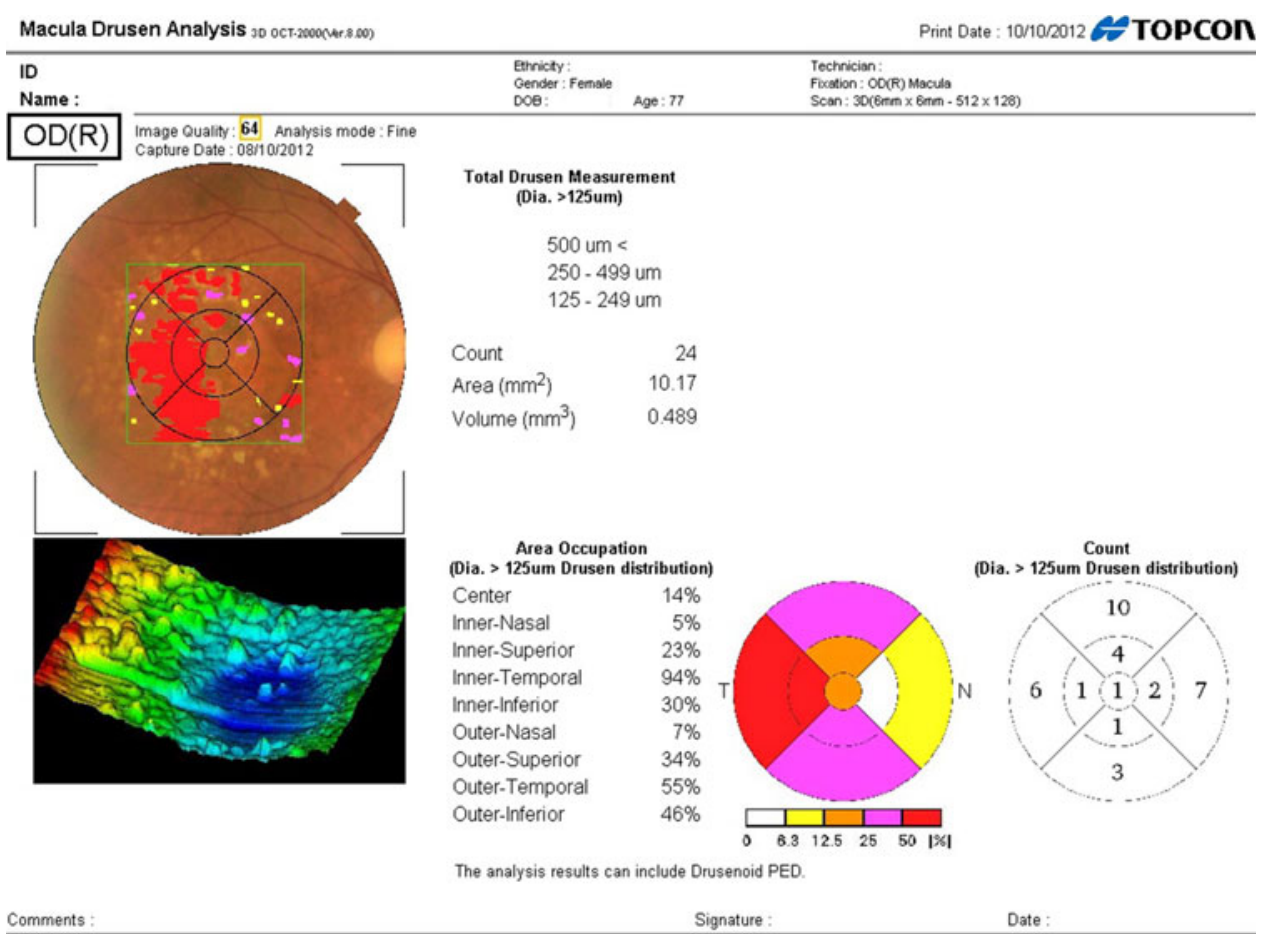

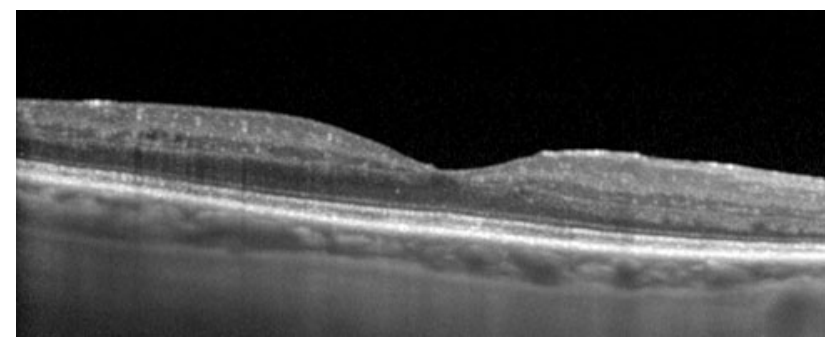

Fig. 6 Enhanced depth imaging (EDI) scanning protocols allow noninvasive visualization of the choroid using optical coherence tomography (OCT) (Spectralis, Heidelberg Engineering)

$12 \times 8 \mathrm{~mm}$ for DRI-OCT, Topcon, versus $6 \times 6 \mathrm{~mm}$ for 3D OCT, Topcon), and even three-dimensional reconstruction of the entire posterior pole [76, 77].

Swept-source OCT systems are also well suited to the incorporation of "long-wavelength" OCT light sources [78]. To date, commercial OCT instruments have tended to use light sources in the range of 800-900 nm. Although imaging with these light sources provides excellent visualization of the retinal architecture, details of sub-RPE structures and abnormalities are often limited. Acquisition of OCT images with longer wavelength light, in the $1,000 \mathrm{~nm}$ range, increases light penetration for visualization of deeper structures. In patients with AMD, long-wavelength OCT may be of particular use for improved visualization of $\mathrm{CNV}$ lesions, including detailed characterization of fibrovascular and/or serous PED. Use of long-wavelength OCT for sub-RPE visualization may also aid phenotyping of drusen and choroidal morphologic disturbances in patients with dry AMD.

\section{Conclusions and Future Directions}

With rapid advances in both diagnosis and treatment, it is an exciting time for those involved in the care of patients with AMD. Advances in anti-angiogenic therapies for neovascular AMD have been accompanied by rapid evolution of OCT imaging. In the near future, this progress is likely to continue, with use of OCT for noninvasive mapping of the retinal and choroidal vasculature ("phase contrast" OCT or "optical coherence angiography") and for quantification of retinal and choroidal blood flow ("Doppler OCT") [79, 80]. Developments in non-neovascular AMD are similarly occurring in tandem with imaging advances. While no definitive treatment yet exists for patients with geographic atrophy, many promising pharmacotherapies are currently in early phase clinical trials [81]. The success of any such approaches is likely to require continued improvement in our imaging capabilities, particularly in the area of adaptive optics, to allow for robust cellular-level assessments, but also with regard to better integration of structural and psychophysical parameters. Although many technical hurdles remain, it is likely that advances in AMD imaging will continue at an exponential rate in the coming years.

Disclosure P.A. Keane and D.A. Sim have received a proportion of their funding from the National Institute for Health Research (NIHR) Biomedical Research Centre based at Moorfields Eye Hospital NHS Foundation Trust and UCL Institute of Ophthalmology. The views expressed are those of the author(s) and not necessarily those of the NHS, the NIHR or the Department of Health; S.R. Sadda is a coinventor of Doheny intellectual property related to optical coherence 
tomography that has been licensed by Topcon Medical Systems, and is a member of the scientific advisory board for Heidelberg Engineering. Dr. Sadda also receives research support from Carl Zeiss Meditec, Optos, and Optovue, Inc. He also served as a consultant for Genentech, Inc., Regeneron, and Allergan, Inc.

\section{References}

Papers of particular interest, published recently, have been highlighted as:

• Of major importance

1. Bressler NM. Age-related macular degeneration is the leading cause of blindness. JAMA. 2004;291(15):1900-1.

2. Keane PA, Sadda SR. Imaging chorioretinal vascular disease. Eye (Lond). 2010;24(3):422-7.

3. Novotny HR, Alvis DL. A method of photographing fluorescence in circulating blood in the human retina. Circulation. 1961; 24:82-6.

4. Gass JD. Pathogenesis of disciform detachment of the neuroepithelium. Am J Ophthalmol 1967;63(3):Suppl:1-139.

5. Gass JD. Photocoagulation of macular lesions. Trans Am Acad Ophthalmol Otolaryngol. 1971;75(3):580-608.

6. Laser photocoagulation of subfoveal neovascular lesions of agerelated macular degeneration. Updated findings from two clinical trials. Macular Photocoagulation Study Group. Arch Ophthalmol. 1993;111(9):1200-9.

7. Connell PP, Keane PA, O’Neill EC, et al. Risk factors for agerelated maculopathy. J Ophthalmol. 2009;2009:360764.

8. • Jager RD, Mieler WF, Miller JW. Age-related macular degeneration. N Engl J Med. 2008;358(24):2606-17. This paper provides an excellent introduction to the $A M D$, with well written descriptions of disease course, classification and current management.

9. von Helmholtz H. Beschreibung eines Augenspiegels zur Untersuchung der Netzhaut in lebenden Auge. Berlin: Germany; 1851.

10. Bennett TJ, Barry CJ. Ophthalmic imaging today: an ophthalmic photographer's viewpoint-a review. Clin Exp Ophthalmol. 2009;37(1):2-13.

11. • Yannuzzi LA, Ober MD, Slakter JS, et al. Ophthalmic fundus imaging: today and beyond. Am J Ophthalmol 2004;137(3): 511-24. This article provides an excellent review of developments in ocular imaging, particularly with regard to areas of imaging other than OCT. It's particular strength is the historical context within which the authors place each new imaging technique.

12. Bernardes R, Serranho P, Lobo C. Digital ocular fundus imaging: a review. Ophthalmologica. 2011;226(4):161-81.

13. Bastawrous A. Smartphone fundoscopy. Ophthalmology 2012;119(2):432-3 e2; author reply 3.

14. Chew EY, Clemons T, Sangiovanni JP, et al. The Age-related Eye Disease Study 2 (AREDS 2): Study Design and Baseline Characteristics (AREDS 2 Report Number 1). Ophthalmology. 2012;119(11):2282-9.

15. Webb RH, Hughes GW, Pomerantzeff O. Flying spot TV ophthalmoscope. Appl Opt. 1980;19(17):2991-7.

16. Strouthidis NG, Garway-Heath DF. New developments in Heidelberg retina tomograph for glaucoma. Curr Opin Ophthalmol. 2008;19(2):141-8.

17. Yamamoto M, Mizukami S, Tsujikawa A, et al. Visualization of cystoid macular oedema using a scanning laser ophthalmoscope in the retro-mode. Clin Exp Ophthalmol. 2010;38(1):27-36.
18. Pilotto E, Sportiello P, Alemany-Rubio E, et al. Confocal scanning laser ophthalmoscope in the retromode imaging modality in exudative age-related macular degeneration. Graefes Arch Clin Exp Ophthalmol. 2013;251(1):27-34.

19. Shin YU, Lee BR, Kim S, Lee WJ. A novel noninvasive detection method for retinal nonperfusion using confocal red-free imaging. Ophthalmology. 2012;119(7):1447-54.

20. Charbel Issa P, Berendschot TT, Staurenghi G, et al. Confocal blue reflectance imaging in type 2 idiopathic macular telangiectasia. Invest Ophthalmol Vis Sci. 2008;49(3):1172-7.

21. Sadda SR, Liakopoulos S, Keane PA, et al. Relationship between angiographic and optical coherence tomographic (OCT) parameters for quantifying choroidal neovascular lesions. Graefes Arch Clin Exp Ophthalmol. 2010;248(2):175-84.

22. Barbazetto I, Burdan A, Bressler NM, et al. Photodynamic therapy of subfoveal choroidal neovascularization with verteporfin: fluorescein angiographic guidelines for evaluation and treatment-TAP and VIP report No. 2. Arch Ophthalmol. 2003;121(9):1253-68.

23. Schmidt-Erfurth U, Teschner S, Noack J, Birngruber R. Threedimensional topographic angiography in chorioretinal vascular disease. Invest Ophthalmol Vis Sci. 2001;42(10):2386-94.

24. Bernardes R, Santos T, Serranho P, et al. Noninvasive evaluation of retinal leakage using optical coherence tomography. Ophthalmologica. 2011;226(2):29-36.

25. Desmettre T, Devoisselle JM, Mordon S. Fluorescence properties and metabolic features of indocyanine green (ICG) as related to angiography. Surv Ophthalmol. 2000;45(1):15-27.

26. Stanga PE, Lim JI, Hamilton P. Indocyanine green angiography in chorioretinal diseases: indications and interpretation: an evidence-based update. Ophthalmology 2003;110(1):15-21; quiz 2-3.

27. Machemer R, Norton EW, Gass JD, Choromokos E. Pseudofluorescence - a problem in interpretation of fluorescein angiograms. Am J Ophthalmol. 1970;70(1):1-10.

28. • Schmitz-Valckenberg S, Holz FG, Bird AC, Spaide RF. Fundus autofluorescence imaging: review and perspectives. Retina 2008;28(3):385-409. This review, written by leading experts in the field, provides everything you could ever wish to know with regard to fundus autofluorescence imaging. It is particularly strong in describing the evolution of this modality, and in the interpretation of FAF images.

29. Delori FC. Spectrophotometer for noninvasive measurement of intrinsic fluorescence and reflectance of the ocular fundus. Appl Opt. 1994;33(31):7439-52.

30. Delori FC, Dorey CK, Staurenghi G, et al. In vivo fluorescence of the ocular fundus exhibits retinal pigment epithelium lipofuscin characteristics. Invest Ophthalmol Vis Sci. 1995;36(3):718-29.

31. Weiter JJ, Delori FC, Wing GL, Fitch KA. Retinal pigment epithelial lipofuscin and melanin and choroidal melanin in human eyes. Invest Ophthalmol Vis Sci. 1986;27(2):145-52.

32. von Ruckmann A, Fitzke FW, Bird AC. Distribution of fundus autofluorescence with a scanning laser ophthalmoscope. Br J Ophthalmol. 1995;79(5):407-12.

33. Hassenstein A, Meyer CH. Clinical use and research applications of Heidelberg retinal angiography and spectral-domain optical coherence tomography-a review. Clin Exp Ophthalmol. 2009;37(1):130-43.

34. Spaide RF. Fundus autofluorescence and age-related macular degeneration. Ophthalmology. 2003;110(2):392-9.

35. Sharifzadeh M, Bernstein PS, Gellermann W. Nonmydriatic fluorescence-based quantitative imaging of human macular pigment distributions. J Opt Soc Am A Opt Image Sci Vis. 2006;23(10):2373-87.

36. Degli Esposti S, Egan C, Bunce C, et al. Macular pigment parameters in patients with macular telangiectasia (MacTel) and 
normal subjects: implications of a novel analysis. Invest Ophthalmol Vis Sci. 2012;53(10):6568-75.

37. Keilhauer CN, Delori FC. Near-infrared autofluorescence imaging of the fundus: visualization of ocular melanin. Invest Ophthalmol Vis Sci. 2006;47(8):3556-64.

38. Kellner S, Kellner U, Weber BH, et al. Lipofuscin- and melaninrelated fundus autofluorescence in patients with ABCA4-associated retinal dystrophies. Am J Ophthalmol 2009;147(5):895-902, e1.

39. Kellner S, Weinitz S, Kellner U. Spectral domain optical coherence tomography detects early stages of chloroquine retinopathy similar to multifocal electroretinography, fundus autofluorescence and near-infrared autofluorescence. $\mathrm{Br} \mathrm{J}$ Ophthalmol. 2009;93(11):1444-7.

40. Schmitz-Valckenberg S, Fleckenstein M, Scholl HP, Holz FG. Fundus autofluorescence and progression of age-related macular degeneration. Surv Ophthalmol. 2009;54(1):96-117.

41. Bearelly S, Cousins SW. Fundus autofluorescence imaging in age-related macular degeneration and geographic atrophy. Adv Exp Med Biol. 2010;664:395-402.

42. Delori FC, Fleckner MR, Goger DG, et al. Autofluorescence distribution associated with drusen in age-related macular degeneration. Invest Ophthalmol Vis Sci. 2000;41(2):496-504.

43. Holz FG, Bindewald-Wittich A, Fleckenstein M, et al. Progression of geographic atrophy and impact of fundus autofluorescence patterns in age-related macular degeneration. Am J Ophthalmol. 2007;143(3):463-72.

44. Staurenghi G, Viola F, Mainster MA, et al. Scanning laser ophthalmoscopy and angiography with a wide-field contact lens system. Arch Ophthalmol. 2005;123(2):244-52.

45. Reeves GM, Kumar N, Beare NA, Pearce IA. Use of Staurenghi lens angiography in the management of posterior uveitis. Acta Ophthalmol. 2013;91(1):48-51.

46. Shields CL, Materin M, Shields JA. Panoramic imaging of the ocular fundus. Arch Ophthalmol. 2003;121(11):1603-7.

47. Wessel MM, Aaker GD, Parlitsis G, et al. Ultra-wide-field angiography improves the detection and classification of diabetic retinopathy. Retina. 2012;32(4):785-91.

48. Wessel MM, Nair N, Aaker GD, et al. Peripheral retinal ischaemia, as evaluated by ultra-widefield fluorescein angiography, is associated with diabetic macular oedema. $\mathrm{Br} \mathrm{J}$ Ophthalmol. 2012;96(5):694-8.

49. Silva PS, Cavallerano JD, Sun JK, et al. Nonmydriatic Ultrawide-field retinal imaging compared with dilated standard 7-field $35-\mathrm{mm}$ photography and retinal specialist examination for evaluation of diabetic retinopathy. Am J Ophthalmol. 2012;154(3): $549-59$ e2.

50. Witmer MT, Cho M, Favarone G, et al. Ultra-wide-field autofluorescence imaging in non-traumatic rhegmatogenous retinal detachment. Eye (Lond). 2012;26(9):1209-16.

51. Heussen FM, Tan CS, Sadda SR. Prevalence of peripheral abnormalities on ultra-widefield greenlight $(532 \mathrm{~nm})$ autofluorescence imaging at a tertiary care center. Invest Ophthalmol Vis Sci. 2012;53(10):6526-31.

52. • Williams DR. Imaging single cells in the living retina. Vis Res 2011;51(13):1379-96. The single best article on the emerging field of adaptive optics-well written, informative, and enjoyable to read.

53. Kitaguchi Y, Fujikado T, Bessho K, et al. Adaptive optics fundus camera to examine localized changes in the photoreceptor layer of the fovea. Ophthalmology. 2008;115(10):1771-7.

54. Chen DC, Jones SM, Silva DA, Olivier SS. High-resolution adaptive optics scanning laser ophthalmoscope with dual deformable mirrors. J Opt Soc Am A Opt Image Sci Vis. 2007;24(5):1305-12.

55. Doble N, Choi SS, Codona JL, et al. In vivo imaging of the human rod photoreceptor mosaic. Opt Lett. 2011;36(1):31-3.
56. Roorda A, Zhang Y, Duncan JL. High-resolution in vivo imaging of the RPE mosaic in eyes with retinal disease. Invest Ophthalmol Vis Sci. 2007;48(5):2297-303.

57. Talcott KE, Ratnam K, Sundquist SM, et al. Longitudinal study of cone photoreceptors during retinal degeneration and in response to ciliary neurotrophic factor treatment. Invest Ophthalmol Vis Sci. 2011;52(5):2219-26.

58. •- Drexler W, Fujimoto JG. State-of-the-art retinal optical coherence tomography. Prog Retinal Eye Res. 2008;27(1):45-88. A comprehensive look at every aspect of ophthalmic OCT imaging, with an emphasis on the underlying technologies.

59. Huang D, Swanson EA, Lin CP, et al. Optical coherence tomography. Science. 1991;254(5035):1178-81.

60. • Keane PA, Patel PJ, Liakopoulos S, et al. Evaluation of agerelated macular degeneration with optical coherence tomography. Surv Ophthalmol. 2012;57(5):389-414. A major review covering every aspect of $O C T$ interpretation for patients with AMD.

61. Wojtkowski M, Bajraszewski T, Gorczynska I, et al. Ophthalmic imaging by spectral optical coherence tomography. Am J Ophthalmol. 2004;138(3):412-9.

62. Keane PA, Bhatti RA, Brubaker JW, et al. Comparison of clinically relevant findings from high-speed fourier-domain and conventional time-domain optical coherence tomography. Am J Ophthalmol. 2009;148(2):242-8 e1.

63. Schmidt-Erfurth U, Leitgeb RA, Michels S, et al. Three-dimensional ultrahigh-resolution optical coherence tomography of macular diseases. Invest Ophthalmol Vis Sci. 2005;46(9):3393-402.

64. Yehoshua Z, Rosenfeld PJ, Gregori G, et al. Progression of geographic atrophy in age-related macular degeneration imaged with spectral domain optical coherence tomography. Ophthalmology. 2011;118(4):679-86.

65. Lujan BJ, Rosenfeld PJ, Gregori G, et al. Spectral domain optical coherence tomographic imaging of geographic atrophy. Ophthalmic Surg Lasers Imaging Off $\mathbf{J}$ Int Soc Imaging Eye. 2009;40(2):96-101.

66. Hee MR, Puliafito CA, Wong C, et al. Quantitative assessment of macular edema with optical coherence tomography. Arch Ophthalmol. 1995;113(8):1019-29.

67. Hee MR, Puliafito CA, Duker JS, et al. Topography of diabetic macular edema with optical coherence tomography. Ophthalmology. 1998;105(2):360-70.

68. Gregori G, Wang F, Rosenfeld PJ, et al. Spectral domain optical coherence tomography imaging of drusen in nonexudative age-related macular degeneration. Ophthalmology. 2011;118(7):1373-9.

69. Penha FM, Rosenfeld PJ, Gregori G, et al. Quantitative imaging of retinal pigment epithelial detachments using spectral-domain optical coherence tomography. Am J Ophthalmol. 2012;153(3): $515-23$.

70. Lee SY, Stetson PF, Ruiz-Garcia H, et al. Automated characterization of pigment epithelial detachment by optical coherence tomography. Invest Ophthalmol Vis Sci. 2012;53(1):164-70.

71. Spaide RF, Koizumi H, Pozzoni MC. Enhanced depth imaging spectral-domain optical coherence tomography. Am J Ophthalmol. 2008;146(4):496-500.

72. Manjunath V, Goren J, Fujimoto JG, Duker JS. Analysis of choroidal thickness in age-related macular degeneration using spectral-domain optical coherence tomography. Am J Ophthalmol. 2011;152(4):663-8.

73. Spaide RF. Enhanced depth imaging optical coherence tomography of retinal pigment epithelial detachment in age-related macular degeneration. Am J Ophthalmol. 2009;147(4):644-52.

74. Spaide RF. Age-related choroidal atrophy. Am J Ophthalmol. 2009;147(5):801-10.

75. van Velthoven ME, Faber DJ, Verbraak FD, et al. Recent developments in optical coherence tomography for imaging the retina. Prog Retinal Eye Res. 2007;26(1):57-77. 
76. Hirata M, Tsujikawa A, Matsumoto A, et al. Macular choroidal thickness and volume in normal subjects measured by sweptsource optical coherence tomography. Invest Ophthalmol Vis Sci. 2011;52(8):4971-8.

77. Ellabban AA, Tsujikawa A, Matsumoto A, et al. Three-dimensional tomographic features of dome-shaped macula by sweptsource optical coherence tomography. Am J Ophthalmol. 2013; 155(2):320-328.e2.

78. Keane PA, Ruiz-Garcia H, Sadda SR. Clinical applications of long-wavelength (1,000-nm) optical coherence tomography. Ophthalmic Surg Lasers Imaging Off J Int Soc Imaging Eye. 2011;42(Suppl):S67-74.
79. Wang Y, Fawzi AA, Varma R, et al. Pilot study of optical coherence tomography measurement of retinal blood flow in retinal and optic nerve diseases. Invest Ophthalmol Vis Sci. 2011;52(2):840-5.

80. Motaghiannezam SM, Koos D, Fraser SE. Differential phasecontrast, swept-source optical coherence tomography at 1,060 nm for in vivo human retinal and choroidal vasculature visualization. J Biomed Opt. 2012;17(2):026011.

81. Zarbin MA, Rosenfeld PJ. Pathway-based therapies for agerelated macular degeneration: an integrated survey of emerging treatment alternatives. Retina. 2010;30(9):1350-67. 